\title{
Anxiolytic Activity of Ultra-diluted Aconitum napellus on Wistar rats
}

\section{Nandini Prashanth Bhat ${ }^{1}$, Anjaneyulu Konuri², Huban Thomas ${ }^{3}$ Vidya Monappa ${ }^{4}$, Hareesh Krishnan5, Prasanna Lokadolalu Chandracharya *6}

${ }^{1}$ Kasturba Medical College Manipal, Manipal Academy of Higher Education, Manipal, India. nandini.bhat@manipal.edu

${ }^{2}$ TATA Medical College, Manipal; Manipal Academy of Higher Education, Jamshedpur, India. anjaneyulu.konuri@manipal.edu

${ }^{3}$ Kasturba Medical College Manipal, Manipal Academy of Higher Education, Manipal, India. huban.thomas@manipal.edu

${ }^{4}$ Kasturba Medical College Manipal, Manipal Academy of Higher Education, Manipal, India. vidya.monappa@manipal.edu

${ }^{5}$ Medical Officer [Homoeopathy], Govt Homoeo Dispensary, Melur, India. drhareeshkrishnan@yahoo.co.in

${ }^{6}$ Kasturba Medical College Manipal, Manipal Academy of Higher Education, Basic Sciences Building, Manipal, India.prasanna.lc@manipal.edu

*prasanna.lc@manipal.edu

https://orcid.org/0000-0002-0737-9573

\begin{abstract}
Background Anxiety disorders are highly prevalent that affect millions of people worldwide. Homeopathic system is gaining popularity due to its efficacy in chronic diseases. Ultra-diluted Aconitum napellus is a homeopathic medicine used by clinicians for treatment of acute anxiety. Aim Objective of this study is to test the efficacy of Aconite on experimental anxiety models of Wistar rats. Methodology 18 Female Wistar rats were divided into Control, Anxiety, and Anxiety + Aconite $(\mathrm{ANX}+\mathrm{ACO})$ groups. Acute stress was induced by restraining the rats for six hours daily for five days in Anxiety and ANX + ACO groups. After five days of stress, a single dose of Aconite 200cH was given to ANX + ACO group through oral gavage, whereas control and anxiety group rats were maintained with a normal diet. Following this, behavioral assessment was done. Histological sections of the hippocampal area were taken and stained with Hematoxylin and Eosin and also Cresyl violet stain. Results It was observed that the Aconite treated group had more number of entries and also the percentage of time spent in the open arm was higher than the stressed anxiety group. Histological study of the brain tissue procured from all three groups was analyzed. Conclusion It was inferred that ultra-diluted homeopathic Aconite has decreased anxiety in Wistar rats which were subjected to acute restrain stress. However, the decrease was not statistically significant to deduce Aconite as an anxiolytic.
\end{abstract}

Keywords: Anxiolytic, Ultradiluted, Homeopathy, Aconite, Anxiety

\section{Introduction}

Anxiety disorders are exceedingly predominant globally and signify a leading purpose for consultation in health care $[1,2]$. Large number of psychotropic drugs for disorders are prescribed in conventional treatment but due to their adverse effects its consumption is limited $[3,4]$. The occurrence of anxiety disorder cases in alternative system of treatment is also ever growing [5]. Homeopathic management one of the alternative systems is shown to be increasingly effective in the treatment of anxiety disorders [6]. Homeopathic system of medicine was established by Samuel Hahnemann in the late 18th century, though comparable conceptions existed earlier $[7,8]$. There is a 
noteworthy body of clinical research comprising randomized clinical trials and meta-analyses of such trials which propose that ultra-diluted medicines used in homeopathy have activities which are curative in nature $[9,10]$. A number of countries include homeopathy in their publicly funded healthcare systems and physiochemical research projects [11,12].

To know the efficacy of different types of anxiolytics several animal models have been developed and described especially in rodents [13]. Pyramidal neurons of Cornu ammonis-3 (CA3) area of hippocampus is known to undergo atrophy in rodents in response to physical or psychological stress $[14,15]$. Previous research shows different constituents of plant and animal origin which have been investigated as anxiolytics in such stress induced rodent prototypes [16]. However, the effects of homeopathic medicines on experimental animal models are lesser known and such studies are rarely conducted. Aconite the choice of an anxiolytic was suggested by homeopathic Materia Medica, reporting a number of neurological and anxiety-related symptoms for this medicine, and in part by pre-existing literature [17]. Aconite is a classic homeopathic medicine for the treatment of patients exhibiting neurological symptoms of anxiety. The main characteristic feature indicating Aconite is physical as well as mental tension. Manifestation of mental tension is in the form of anxiety and fear. Literature suggests that acute anxiety, which is the feature of this deadly poison, will be relieved when dosed ultra-diluted [18].

A double-blinded study on 50 children with post-operative agitation, where Aconite was administered, proved to be effective with $95 \%$ reduced anxiety [19]. Aconite ultra-diluted to $200 \mathrm{cH}$, demonstrated to be useful for orthopaedic patients with post-traumatic anxiety [20]. Although Aconite is used to clinically treat patients with anxiety, its mechanism of action is unknown. Through this study we attempt to provide necessary evidence for homeopathic medicine Aconite by testing its efficacy in stress induced animal models through behavioral assessment and histological analysis of hippocampus.

\section{Materials and Methods}

Animal Model: Healthy female Wistar rats from inbred colony maintained at Central Animal Research Facility (Reg No 94/1999/CPCSEA) were used for the study. 18 adult rats with average weight of 250-300 g were maintained in well ventilated polypropylene cages containing paddy husk. Animals were given food and fresh water ad libitum. The protocol for animal use was approved from the Institutional Animal Ethics Committee. (IAEC/KMC/28/2018). 18 rats were divided into the following three groups Control, Stress induced anxiety (ANX) and Anxiety + Aconite (ANX+ACO).

\section{Experimental Design}

\section{Induction of stress}

The rats of anxiety and treatment group's six animals each were placed within the appropriate restrainer, and the entrance was closed by sliding the door. The dimensions of each compartment of this restrainer were $35 \mathrm{~cm}(\mathrm{~L}) \times 35 \mathrm{~cm}(\mathrm{~B}) \times 30 \mathrm{~cm}(\mathrm{H})$. These rats remained within the restrainer for $6 \mathrm{~h}$ on each day (10.00 AM-4.00 PM) for 5 days. The restrainer with the rats was kept on a table in a room with sufficient ventilation. After $6 \mathrm{~h}$ of stress, rats were released from the restrainer and were kept in their respective home cages. The restrainer was washed and dried after use $[21,22,23]$.

\section{Administration of Homeopathic Drug}

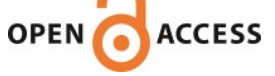


Aconite $200 \mathrm{cH}\left(1 \times 10^{-200}\right)$ high dilution, commercially available from Willmar Schwabe pharmacy, recommended by Homeopathic Materia Medica, AYUSH [24] was administered orally. One 2 oz. vial was taken and filled with $1 \mathrm{oz}$. of distilled or purified water. One medicated globule of size No. 10 of Aconite $200 \mathrm{cH}$ was added to it. It was stirred thoroughly. $1 \mathrm{ml}$ of this solution was given orally to the animal through oral gavage [25].

\section{Behavioral test: The elevated T maze (26)}

This device is in the shape of a cross which is raised to a height of $40 \mathrm{~cm}$ from the floor. The device has two open arms without walls of the size $50 \times 10 \times 59 \mathrm{~cm}$. Perpendicular to the open arms are two other arms closed by walls.

\section{Procedure}

The test takes a total of 5 minutes per animal. The rat was placed in the center of the maze, facing an open arm. Anxious rats are averse to heights and open spaces. A rat exploring the open arms was designated as "less anxious" and a rat that is confined to the closed arms of the device was described as "anxious." Measured variables were a) Entries of the head, nose, and forepaw in the open arm, b) Number of full body entries in the open arm, and c) Percentage of time spent in the open arm [26]. The assessment was done by an unprejudiced observer.

\section{Collection of Brain}

Post-behavioural study, the rats were transcardially perfused with heparinized saline through the left ventricle followed by $10 \%$ formalin at the rate of $1 \mathrm{ml} / \mathrm{min}$ and the brains were fixed with $10 \%$ formalin. Subsequently, these were dehydrated using ascending grades of ethanol and embedded in paraffin wax and blocks were prepared and labelled.

\section{Histological technique}

$5 \mu \mathrm{m}$ thickness sections were cut using rotatory microtome of the prepared tissue blocks. The sections were fixed on slides and stained using Haematoxylin and Eosin (H \& E) according to the protocol given in Principles and Techniques in Histology Microscopy and Photomicrography by Singh et.al [27]. Further sections were also used to stain with cresyl violet. The slides were dipped in the $0.1 \%$ cresyl violet solution for $20-25 \mathrm{~min}$ at $60^{\circ} \mathrm{C}$ in accordance of protocol explained by Shetty et.al [28]. The nucleoproteins and nissel substance is stained using a basic dye, cresyl violet. Also the view detailing the cell bodies can be obtained with the help of this dye.

\section{Histological assessment}

Stained sections were studied under Olympus Research Microscope (model cx21i) and photographed at 10X and 100X magnification. Cornu Ammonis-1(CA1) and Cornu Ammonis-3 (CA3) region of hippocampal formation were considered in at least three random microscopic fields from each animal by an expert pathologist blinded to the treatment groups.

\section{Statistical analysis}

One-way ANOVA followed by Bonferroni's post hoc test using the statistical software, Graph Pad Prism was used for data analysis. Results were expressed as mean \pm SD and p-value $\leq 0.05$ was expressed as significant.

OPEN ACCESS 


\section{Results}

\section{Behavioral assessment}

Twenty-four hours after administration of Aconite $200 \mathrm{cH}$, treatment group animals showed increase in the open arm entry compared to stress group in the elevated plus maze test. The mean \pm SD are as follows, Control- $2 \pm 0.8$, Anx- $0.8 \pm 0.6$, Anx + Aco-1. \pm 0.8 (Figure 1). The forepaw, nose, head entries in the open arm are increased compared to the animals in the stress group. Control- $9.83 \pm$ 1.47, Anx- $5.16 \pm 3.31$, Anx + Aco-10.16 \pm 4.40 (Figure 2). Also the percentage of time spent in the open arm is comparatively increased in the treatment group; Control- $6.16 \pm 5.84$, Anx- $1.45 \pm 2.39$, Anx + Aco- $5.03 \pm 2.5$ (Figure 3). In all these parameters although there is an alteration between the

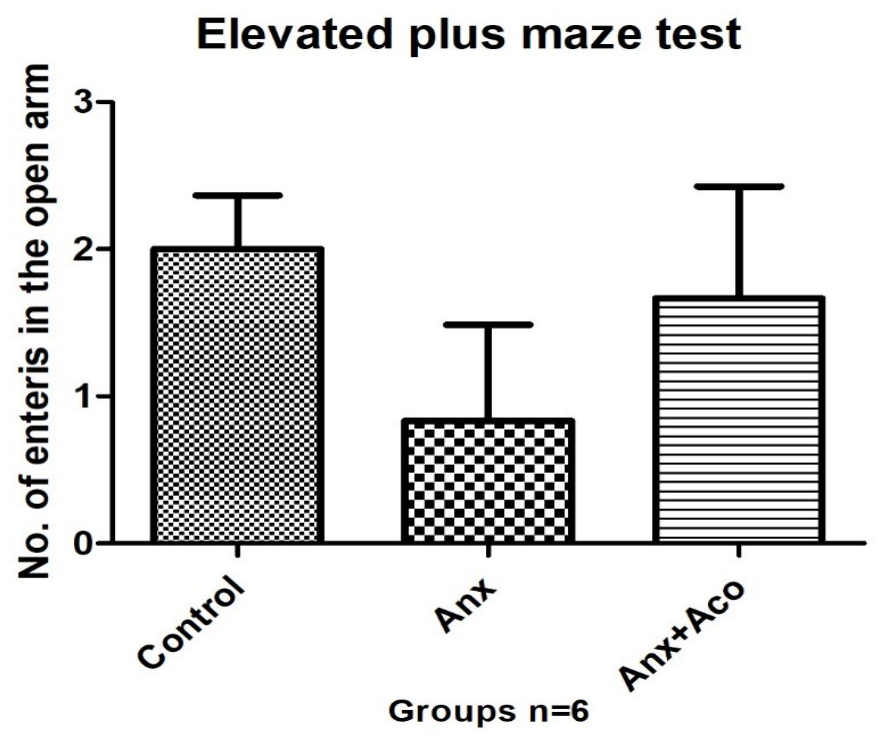
three groups, nevertheless there is no statistical significance $(\mathrm{p}=\geq 0.05)$ seen after applying one way anova followed by Bonferroni's post hoc test.

Figure 1- Effect of Aconite on Behavior of Wistar rats in Elevated plus maze. No. of open arm entries in Control, Stress induced (Anx) and Aconite treated group $($ Anx+Aco).

Figure 2 - Effect of Aconite on Behavior of Wistar rats in Elevated plus maze. No. of the fore paw, head, nose entries in the Control, Stress induced (Anx) and Aconite treated group (Anx+Aco).
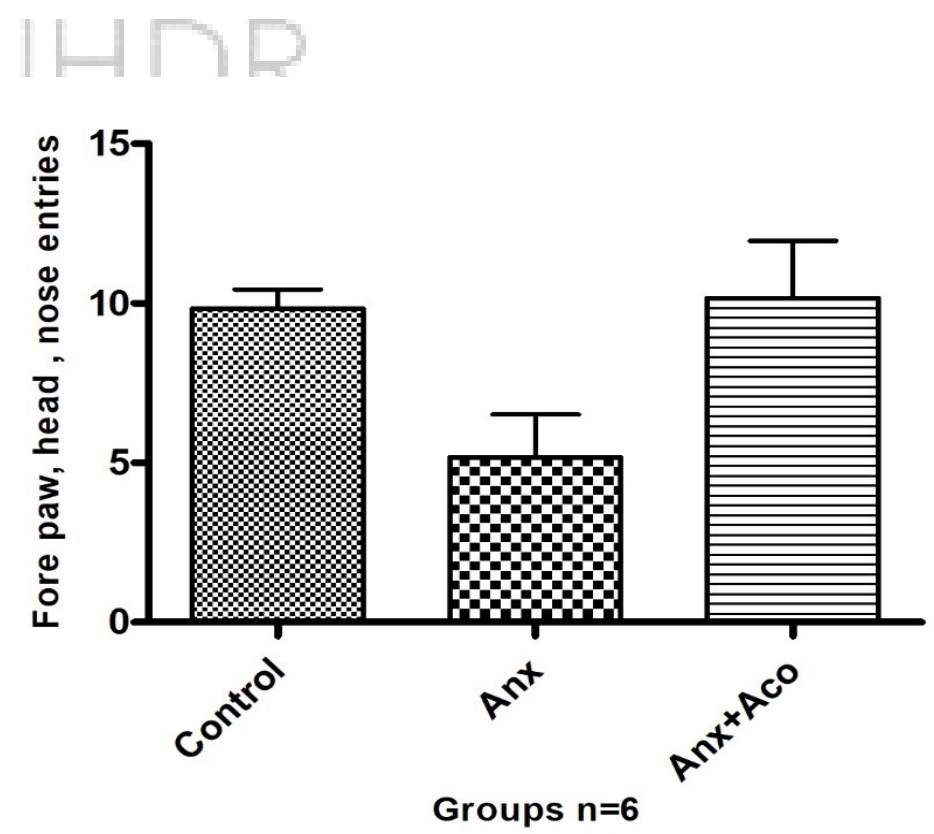


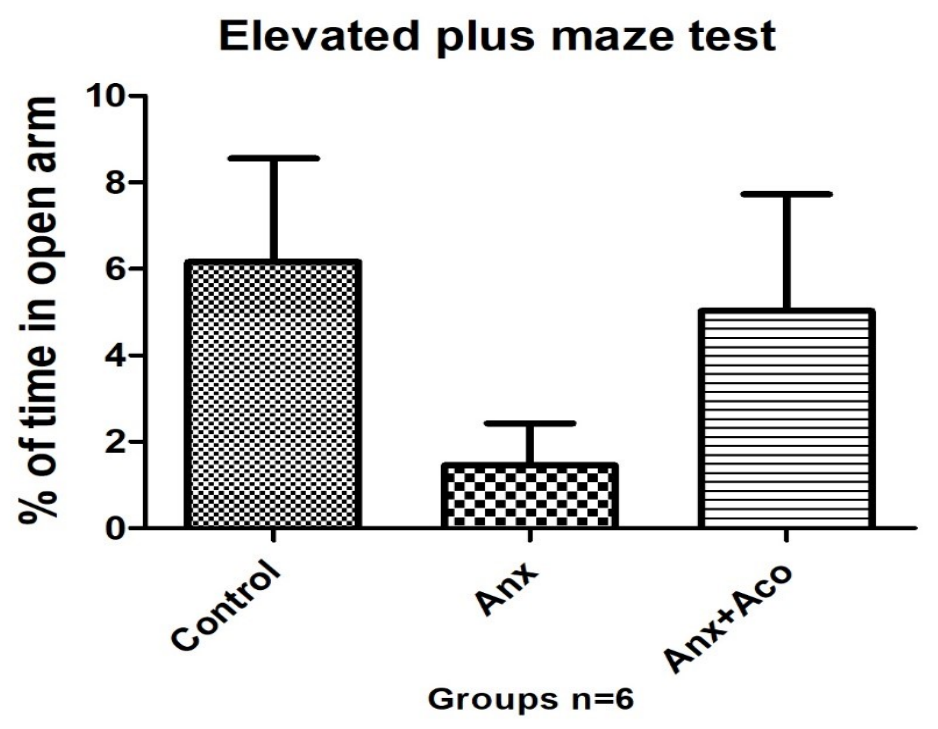

Figure 3-Effect of Aconite on Behavior of Wistar rats in Elevated plus maze. Percentage of time spent in open arm in the Control, Stress induced (Anx) and Aconite treated group (Anx+Aco).

\section{Qualitative analysis of neural cells in Hippocampus}

\section{CA1 region}

Microscopic examination of CA1 sub region of hippocampus showed almost equal number of viable neurons in all three groups. In $\mathrm{H} \& \mathrm{E}$ stain no difference was found in treatment group compared to control and stress group (Figure 4). In cresyl violet stain the number of viable neurons was similar in all three groups (Figure 5). In all three groups, neurons showed clear cytoplasm and distinct nucleus.
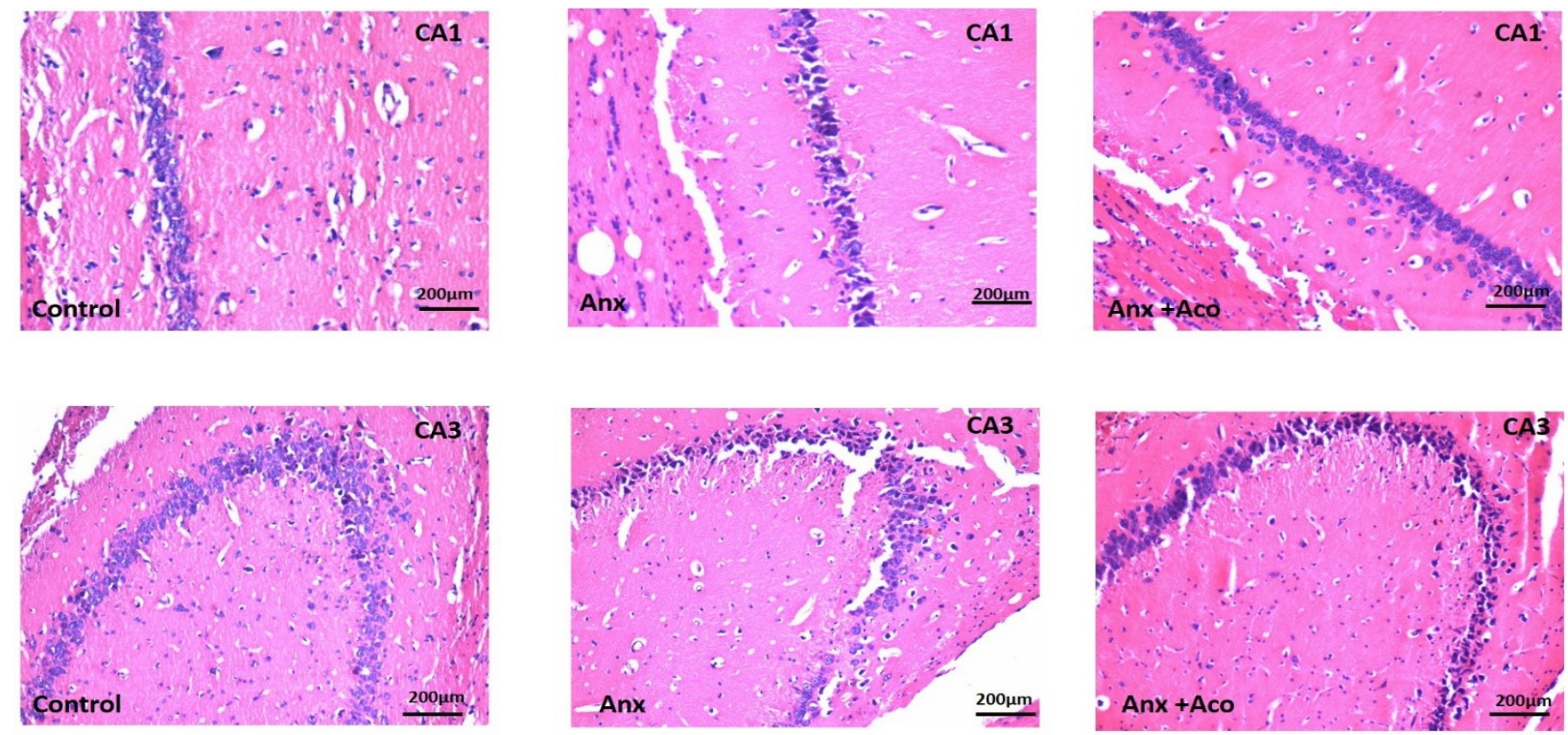

Figure 4 - 10X Haematoxylin and Eosin stain of CA1 and CA3 sub region of the Hippocampus of control, stress induced (Anx) and treatment groups (Anx + Aco). 


\section{CA3 region}

Detailed microscopic examination of the CA3 region too did not show any significant difference in the quality of neurons. In $\mathrm{H} \& \mathrm{E}$ stain all three groups demonstrated similar histological presentation of CA3 region (Figure 4). In cresyl violet stain, the cells appeared similar in all three groups taking uniform stain. With no notable difference between treatment and stress groups (Figure 5).
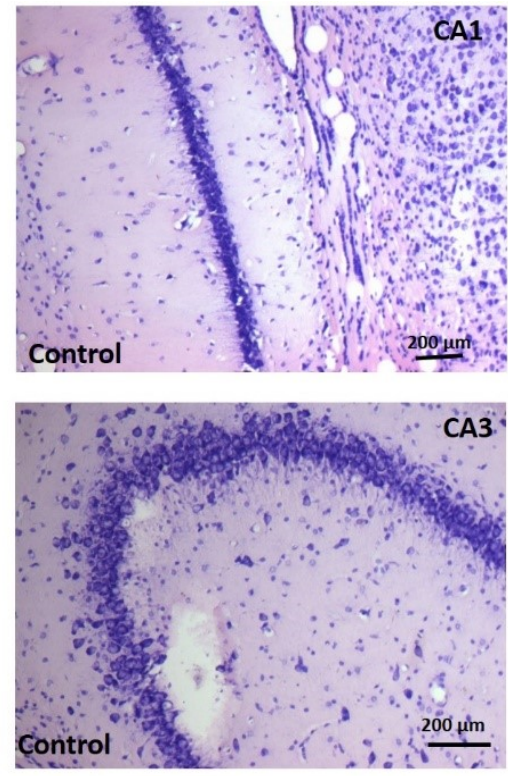
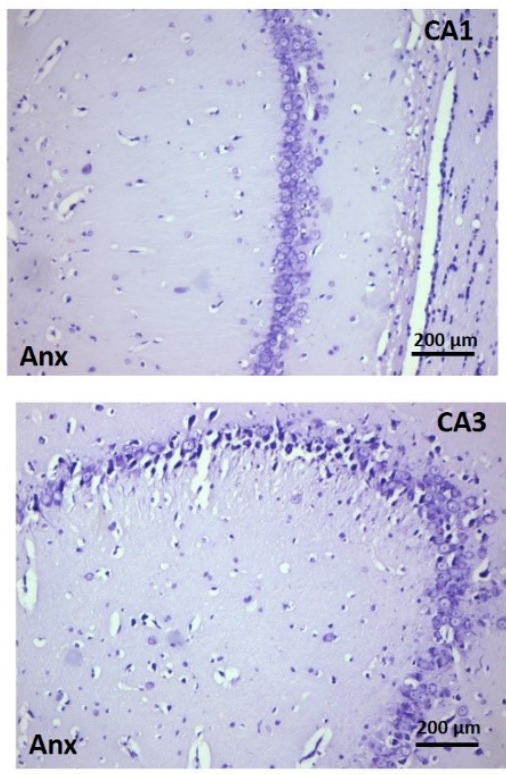
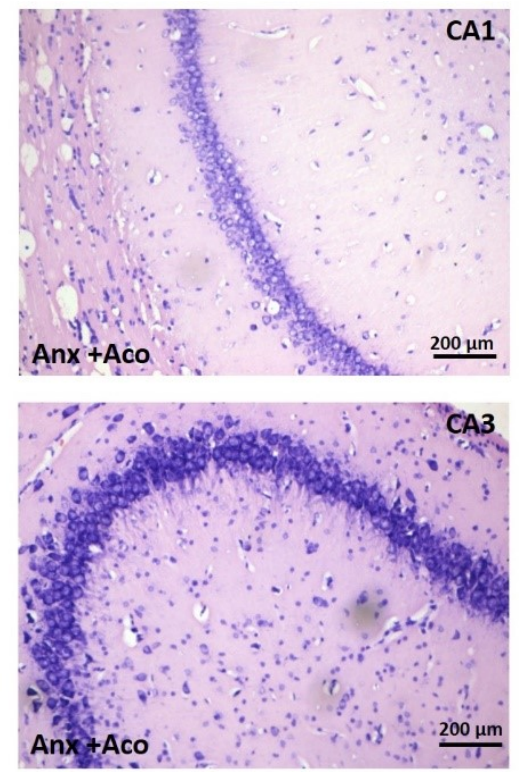

Figure 5-10X Cresyl violet stain of CA1 and CA3 sub region of the Hippocampus of control, stress induced (Anx) and treatment groups (Anx + Aco).

\section{Discussion}

The objective of this study was to obtain behavioral profiles after homeopathic treatment and to evaluate the strengths of ultra-diluted Aconite in experimental anxiety induced rat models. We explored behavioral and histological parameters to come to a conclusion. In the elevated plus maze test the number of entries and time spent in the open arm is suggestively more in treatment group than stress group animals which hints the efficacy of ultra-diluted Aconite $200 \mathrm{cH}$, but cannot be concluded as an anxiolytic as it is not statistically significant. Similar studies done by Haine et al. on Wistar rats, ultra-dilutions of homeopathic $12 \mathrm{cH}$ and $30 \mathrm{cH}$ of Aconite exhibited anxiolytic effects revealed by improved percentage of time and the number of entries in elevated plus maze open arms [29]. The difference between our experiment and the previous research is that they have used Aconite $12 \mathrm{cH}$ and $30 \mathrm{cH}$ whereas we have used further dilution i.e $200 \mathrm{cH}$.

Bellavite et. al have validated that both low and high dilutions of extract of Gelsemium conventionally used for patients with anxiety disorders, decrease anxiety and panic in confirmed mice behavioral models [30,31]. Similar anxiolytic activities have been demonstrated by another Homeopathic remedy Ignatia in ultra-diluted $3 \mathrm{cH}$ and $9 \mathrm{cH}$ potency on experimental rat models. These researchers conclude that ultra-diluted homeopathic medicines act more than placebo and efficacy is shown in anxiety induced rat models [32].

A previous study conducted by researchers showed an anxiolytic effect of homeopathic preparation of Pulsatilla nigricans 3X on animal model comparable to that found with a psychotropic standard 
drug diazepam [33]. Similarly, Argentum nitricum $30 \mathrm{cH}$ a homeopathic drug was found to possess a potent anxiolytic activity in two species rats and mice in comparison with the standard drug escitalopram, and it is suggested that high dilutions of Argentum nitricum should be administered because of its potential to produce toxic effects [34].

There are confirmed reports in the literature of chronic stress causing apoptosis in hippocampal region as well as cerebral cortex $[35,36]$. There are numerous variances seen in studies due to differences in the species of rodents, type and duration of stress, housing conditions, type of behavioral tests etc. Recognition of stress in laboratory animal models necessitates an understanding of these inconsistencies. [37, 38]

In our research according to the protocol we have observed changes in behavioral study. However, we haven't perceived any noteworthy changes in histology of brain, with no significant neuronal death in hippocampal region. The reason for this maybe that the stress induced on the rats were for short duration of $6 \mathrm{~h}$ for 5 days. It was not sufficient to cause any pathological changes in the brain as demonstrated by the normal architecture of the CA1 and CA3 areas of the hippocampus.

\section{Conclusion}

In the present study we evaluated the efficacy of ultra-diluted Aconitum napellus as an anxiolytic. We conclude that though Aconite shows some anxiolytic activity, it does not appear to be significant as demonstrated by the results of the behavioral tests in the stress induced rats. Further studies with diverse experimental stress models need to be done on large number of animals with immunohistochemistry test for fortification of its anxiolytic action.

\section{References}

[1] Vos T, Flaxman AD, Naghavi M, Lozano R, Michaud C. Years lived with disability (YLDs) for 1160 sequelae of 289 diseases and injuries 1990-2010: a systematic analysis for the Global Burden of Disease Study 2010. Lancet 2012; 380 (9859): 2163-96. doi: 10.1016/S0140-6736(12)61729-2.

[2] Grimaldi-Bensouda L, Begaud B, Lert F, Rouillon F, Massol J Guillemot D, Avouac B, Duru G, Magnier A, Rossignol M, Abenhaim L. Benchmarking the burden of 100 diseases: results of a nationwide representative survey within general practices. British Medical Journal Open 2011; 1 (2): e000215-e000215. doi: 10.1136/bmjopen-2011-000215

[3] Fournier J, DeRubeis R, Hollon S, Dimidjian S, Amsterdam J, Shelton R, Fawcett J. Antidepressant Drug Effects and Depression Severity. The Journal of the American Medical Association 2010; 303 (1): 47. doi: 10.1001/jama.2009.1943

[4] Hefner G, Stieffenhofer V, Gabriel S, Palmer G, Müller K, Röschke J, Hiemke C. Side effects related to potentially inappropriate medications in elderly psychiatric patients under everyday pharmacotherapy. European Journal of Clinical Pharmacology 2014; 71 (2): 165-172. doi: 10.1007/s00228-014-1796-5

[5] Gelabert R. Clinical Study of the Effectiveness of Pluralist Homeopathy in Generalized Anxiety Disorder. Alternative \& Integrative Medicine 2017; 06 (04). doi: 10.4172/23275162.1000250 
[6] Mathie R, Fok Y, Viksveen P, To A, Davidson J. Systematic Review and Meta-Analysis of Randomised, Other-than-Placebo Controlled, Trials of Non-Individualised Homeopathic Treatment. Homeopathy 2019; 108 (02): 088-101. doi: 10.1055/s-0038-1677481

[7] Fisher P. What is Homeopathy An Introduction. Frontiers in Bioscience 2012; E4 (5): 1669-1682. doi: $10.2741 / \mathrm{e} 489$

[8] Milgrom LR. 2006. Is homeopathy possible? Journal of the Royal Society for the Promotion of Health 2006; 126 (5): 211-218. doi:10.1177/1466424006068237

[9] Mathie R, Lloyd S, Legg L, Clausen J, Moss S, Davidson J, Ford I. Randomised placebocontrolled trials of individualised homeopathic treatment: systematic review and metaanalysis. Systematic Reviews 2014; 3(1). doi: 10.1186/2046-4053-3-142.

[10] Ive E, Couchman I, Reddy L. Therapeutic Effect of Arsenicum album on Leukocytes. International Journal of Molecular Sciences 2012; 13 (3): 3979-3987. doi:10.3390/ijms13033979

[11] Brenes-Valverde A. Effectiveness of homeopathic treatment. British Homeopathic Journal 2000; 89 (s1): S54-S54. doi: 10.1038/sj.bhj.5800393

[12] Klein S, Würtenberger S, Wolf U, Baumgartner S, Tournier A. Physicochemical Investigations of Homeopathic Preparations: A Systematic Review and Bibliometric Analysis-Part 1. Journal of Alternative and Complementary Medicine 2018; 24 (5):409421. doi: $10.1089 / \mathrm{acm} .2017 .0249$

[13] Bourin M. Animal models for screening anxiolytic-like drugs: a perspective. Dialogues Clinical Neuroscience 2015; 17 (3): 295-303.

[14] McEwen B. Neurobiological and Systemic Effects of Chronic Stress. Chronic Stress 2017; 1:24. doi: 10.1177/2470547017692328

[15] Kim E, Pellman B, Kim J. Stress effects on the hippocampus: a critical review. Learning and Memory 2015; 22 (9): 411-416. doi: 10.1101/lm.037291.114

[16] McIntyre E, Saliba A, Wiener K, Sarris J. Herbal medicine use behaviour in Australian adults who experience anxiety: a descriptive study. BMC Complementary Alternative Medicine 2016; 16(1). doi: 10.1186/s12906-016-1022-3

[17] Clarke JH. Dictionary of materia medica: A dictionary of practical materia medica. Essex, England: Health Science Press. 1982

[18] Kent J. Lectures on homoeopathic materia medica. New Delhi: Homeopathic Publications; 1994.

[19] Alibeu JP, Jobert J. Aconite in homeopathic relief of post-operative pain and agitation in children. Pediatrie 1990; 45 (7-8), 465-466.

[20] Oberbaum M, Schreiber R, Rosenthal C, Itzchaki M. Homeopathic treatment in emergency medicine: a case series. Homeopathy 2003; 92 (1): 44-47. doi: 10.1054/homp.2002.0071

[21] Sanger D. Animal Models of Anxiety and the Screening and Development of Novel Anxiolytic Drugs. Animal Models in Psychiatry, II. :147-198.21. doi: 10.1016/02785846(91)90082-c

[22] Kumar K, Sharma S, Kumar P, Deshmukh R. Therapeutic potential of GABAB receptor ligands in drug addiction, anxiety, depression and other CNS disorders. Pharmacology Biochemistry and Behavior 2013; 110,174-184. doi: 10.1016/j.pbb.2013.07.003 
[23] Varlinskaya E, Spear L. Increases in anxiety-like behavior induced by acute stress are reversed by ethanol in adolescent but not adult rats. Pharmacology Biochemistry and Behavior 2012; 100 (3): 440-450. doi: 10.1016\%2Fj.pbb.2011.10.010

[24] R F D - Ministry of AYUSH. (n.d.). Retrieved August 28, 2019 from http://ayush.gov.in/sites/default/files/2822863133-rfd final 1.pdf

[25] Homoeopathic Pharmacopoeia of India, ccrhindia.nic.in/index1.aspx?lsid= 665\&lev=1\&lid=600\&langid=1. Retrived Oct $11^{\text {th }}, 2019$

[26] Pellow S, Chopin P, File S, Briley M. Validation of open: closed arm entries in an elevated plus-maze as a measure of anxiety in the rat. The Journal of Neuroscience Methods 1985; 14 (3): 149-167. doi: 10.1016/0165-0270(85)90031-7

[27] Singh DR, Principles and Techniques in Histology Microscopy and Photomicrography Published by CBS Publishers and Distributors, $1^{\text {st }}$ edition, pg \# 20-40, 2003.

[28] Shetty A, Turner D. Fetal Hippocampal Grafts Containing CA3 Cells Restore Host Hippocampal Glutamate Decarboxylase-Positive Interneuron Numbers in a Rat Model of Temporal Lobe Epilepsy. Journal of Neuroscience 2000; 20 (23): 8788-8801. doi: 10.1523\%2FJNEUROSCI.20-23-08788.2000

[29] Haine G.B. Assessment of homeopathic medicine Aconitum napellus in the treatment of anxiety in an animal model. International Journal of High Dilution Research 2012; 11: 33-42.

[30] Bellavite P, Magnani P, Zanolin E, Conforti A. Homeopathic Doses of Gelsemium sempervirens Improve the Behavior of Mice in Response to Novel Environments. Evidence Based Complementary and Alternative Medicine 2011; 2011:1-10. doi: 10.1093/ecam/nep139

[31] Bellavite P, Conforti A, Marzotto M, Magnani P, Cristofoletti M, Olioso D, Zanolin M. Testing Homeopathy in Mouse Emotional Response Models: Pooled Data Analysis of Two Series of Studies. Evidence Based Complementary and Alternative Medicine 2012; 2012: 19. doi: 10.1155/2012/954374

[32] Marzotto M, Conforti A, Magnani P, Zanolin M, Bellavite P. Effects of Ignatia amara in mouse behavioural models. Homeopathy 2012; 101 (1): 57-67. doi: 10.1016/j.homp.2011.10.001

[33] Lakshmipathy PR, Ruckmani A, Venkatesan D, Madhusudhanan N, Pavithra R. Anxiolytic effect of homeopathic preparation of Pulsatilla nigricans in Swiss albino mice. Homeopathy 2012; 101 (3): 171-174. doi: 10.1016/j.homp.2012.05.003

[34] Anser H, Najam R, Khatoon H, Khan S. Evaluation Of Anxiolytic Activity Of Homeopathic Remedy Argentum Nitricum Of 30 C Potency After Chronic Administration In Rodents International Journal of Pharma Sciences and Research 2018; Vol.9 (9): 39083913. doi: 10.13040/IJPSR.0975-8232.9(9).3908-13

[35] Lucassen P, Vollmann-Honsdorf G, Gleisberg M, Czéh B, De Kloet E, Fuchs E. Chronic psychosocial stress differentially affects apoptosis in hippocampal subregions and cortex of the adult tree shrew. European Journal of Neuroscience 2001; 14 (1): 161-166. doi: 10.1046/j.0953-816x.2001.01629.x

[36] McLaughlin K, Gomez J, Baran S, Conrad C. The effects of chronic stress on hippocampal morphology and function: An evaluation of chronic restraint paradigms. Brain Research 2007; 1161: 56-64. doi: 10.1016/j.brainres.2007.05.042 
[37] Animals, N., 2020. Recognition And Assessment Of Stress And Distress. [online] Ncbi.nlm.nih.gov. Available at: <https://www.ncbi.nlm.nih.gov/books/NBK4033/> [Accessed 28 April 2020]

[38] Ducottet C, Belzung C. Correlations between behaviours in the elevated plus-maze and sensitivity to unpredictable subchronic mild stress: evidence from inbred strains of mice. Behavioural Brain Research. 2005; 156(1): 153-162. doi:10.1016/j.bbr.2004.05.018

Received: Feb 04, 2020. Accepted: Apr 05, 2020.

(C) International Journal of High Dilution Research.

Not for commercial purposes.

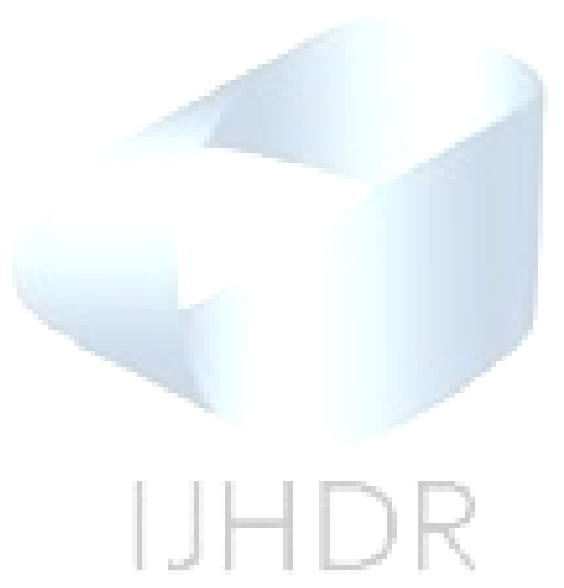

